test. We also show that AD and PD have a statistically significant association with the gait speed and sit-to-stand test performance.

\section{AN ACTIVITY INDEX FOR RAW ACCELEROMETRY DATA AND ITS APPLICATION IN OLDER ADULTS} J. Bai ${ }^{1}$, C. $\mathrm{Di}^{2}$, L. Xiao ${ }^{3}$, K.R. Evenson ${ }^{4}$, A. LaCroix ${ }^{5}$, C. Crainiceanu ${ }^{1}$, D.M. Buchner ${ }^{6}, 1$. Department of Biostatistics, Johns Hopkins University, Baltimore, Maryland, 2. Fred Hutchinson Cancer Research Center, Seattle, Washington, 3. North Carolina State University at Raleigh, Raleigh, North Carolina, 4. University of North Carolina - Chapel Hill, Chapel Hill, North Carolina, 5. University of California, San Diego, La Jolla, California, 6. University of Illinois at Urbana-Champaign, Champaign, Illinois

Accelerometers have been widely deployed in public health studies in recent years and research has mainly focused on summarized metrics provided by accelerometers manufactures, such as the activity counts (AC). Such measures do not have a publicly available formula and can vary by device manufacturer. To address these problems, we developed the activity index (AI), a new metric for summarizing raw triaxial accelerometry data, and compared the AI to AC's performance for distinguishing various types of activities and estimating energy expenditure. The analysis was conducted using data from the Women's Health Initiative, in which triaxial raw acceleration data and energy expenditure were collected at the same time. ROC analyses indicated that AI better distinguished between different types of activities than AC. AI better associated with METs as well. The proposed AI provides a transparent and reliable way to summarize densely sampled raw acceleration data.

\section{PHYSICAL EXERTION AND ACTIVITY: AGE AND RELATIVE EFFORT IN THE BALTIMORE LONGITUDINAL STUDY OF AGING}

A. Leroux ${ }^{1}$, J. Schrack ${ }^{1}$, J. Fleg ${ }^{3}$, E.M. Simonsick ${ }^{2}$, V. Zipunnikov' ${ }^{1}$, S.A. Studenski², L. Ferrucci², C. Crainiceanu ${ }^{1}$, 1. Johns Hopkins University Bloomberg School of Public Health, Baltimore, Maryland, 2. National Institute on Aging, Baltimore, Maryland, 3. National Heart, Lung, and Blood Institute, Bethesda, Maryland

Commonly used measures of physical activity (PA) fail to account for heterogeneity in maximal capacity between individuals. Utilizing the concept of heart rate reserve, we estimated minute level physical exertion (PE) among BLSA subjects with at least 3 days of Actiheart data $(n=411)$. Subjects' average time spent in each of sedentary, light, moderate and vigorous PE was associated with age adjusting for demographics, chronic conditions, and PA. Despite a significant decline in total PA with age $(\mathrm{p}<0.001)$, time spent in moderate and vigorous $\mathrm{PE}$ is positively associated with age in both women $\left(\mathrm{p}_{\text {moderate }}<0.001, \mathrm{p}_{\text {vigorous }}<0.001\right)$ and men $\left(\mathrm{p}_{\text {moderate }}=0.137, \mathrm{p}_{\text {vigorous }}=0.222\right)$. Chronic conditions are also generally associated with increased average PE. Our results suggest that individuals with decreased capacity are, on average, engaging moderate and high relative intensity activities at a rate comparable to, or higher than, that of healthier individuals.

\section{AUTOMATIC CAR DRIVING DETECTION USING RAW ACCELEROMETRY DATA}

M. Straczkiewicz ${ }^{2}$, J. Urbanek ${ }^{3}$, W. Fadel ${ }^{1}$, C. Crainiceanu ${ }^{3}$, J. Harezlak ${ }^{1}, 1$. Biostatistics, Indiana University Fairbanks School of Public Health, Indianapolis, Indiana, 2. AGH University of Science and Technology, Krakow, Poland, 3. Johns Hopkins Bloomberg School of Public Health, Baltimore, Maryland

Measuring physical activity using wearable devices has gained popularity in older populations. Raw data collected from them is summarized as "activity counts", which combine information of human activity with environmental vibrations. Driving is a major sedentary activity that can artificially increase these counts. Thus, it is important to identify periods of driving and quantify the bias induced by driving in activity counts. To address these problems, we propose a Detection Algorithm of Driving via Accelerometry (DADA), which is based on short-time Fourier transform applied to the raw accelerometry data identifying the frequencies' ranges specific to car driving. We test DADA's performance on data collected using wristworn ActiGraph devices in an experiment conducted on 24 subjects. The median AUC for predicting driving periods was 0.94 . The activity count bias induced by driving per unit of time was on average equal to $16 \%$ of the average activity counts generated during walking.

\section{TOTAL PHYSICAL ACTIVITY AND ITS CIRCADIAN ALLOCATION ARE INDEPENDENT PREDICTORS OF MORTALITY}

V. Zipunnikov, D. Dey, A. Leroux, J. Di, J. Urbanek, J. Schrack, C. Crainiceanu, Johns Hopkins Bloomberg School of Public Health, Baltimore, Maryland

We examine whether the distribution of physical activity during the day acts as an independent predictor of mortality in National Health and Nutrition Examination Survey 2003-2006. Time of day analyses were conducted using 12 time windows of 2 hours represented by the percentage of the total log-transformed activity count (pTLAC) spent in each. In models adjusted for age, sex, race/ethnicity, education, body mass index, comorbidities, and the total log-transformed activity count, a higher percentage of total physical activity between 10am-12pm (HR: 0.96, $\mathrm{p}$-value $=0.003)$ and a lower percentage of total physical activity between $8 \mathrm{pm}-4 \mathrm{am}$ (HR: 1.03 , p-value $<0.01$ ) were significantly associated with lower risk of mortality. These findings help define patterns of physical activity that are beneficial to health, and hold significant implications for future interventions aimed at increasing physical activity and enhancing sleep quality.

\section{SESSION 4600 (SYMPOSIUM)}

\author{
IAGG SECRETARIAT: THEORETICAL AND POLICY \\ PERSPECTIVES FOR HEALTHY AGING \\ Chair: Y. Lee, Ajou University School of Medicine, Suwon, \\ Korea (the Republic of) \\ Co-Chair: D. Lee, Seoul National University College of \\ Medicine, Korea (the Republic of)
}

